\title{
Olfactory Glomeruli in the Zebrafish Form an Invariant Pattern and Are Identifiable Across Animals
}

\author{
Herwig Baier and Sigrun Korsching \\ Max-Planck-Institut für Entwicklungsbiologie, Abteilung Physikalische Biologie, D-72011 Tübingen, Germany
}

\begin{abstract}
Glomeruli are anatomical and possibly functional modules in the vertebrate olfactory bulb. We investigated the spatial arrangement of glomeruli in the olfactory bulbs of adult zebratish (Brachydanio rerio). A solution of the lipophilic tracer Dil was injected into the nasal cavities. Axons of sensory neurons projecting from the olfactory epithelium into the bulb were traced anterogradely, thus labeling the whole population of glomeruli. The glomerular distribution was analyzed in detail by confocal laser-scanning microscopy.

We find that a typical olfactory bulb contains a small number of about $\mathbf{8 0}$ glomeruli that have a stereotyped configuration in all animals investigated. All glomeruli exhibit bilateral symmetry. Twenty-two single glomeruli could be identified from animal to animal by their characteristic position and morphology. The remaining glomeruli either are embedded in glomerular plexus and therefore cannot be delineated reliably, or belong to a densely clustered subpopulation of on average $\mathbf{4 9}$ glomeruli in the dorsal olfactory bulb. No sexually dimorphic glomeruli were identified. To test whether glomerular constancy is specific for the zebrafish, we performed similar tracing experiments in the goldfish and found several indications for a similar invariance of glomeruli in this species.
\end{abstract}

The remarkable stereotypy of this pattern is reminiscent of the insect olfactory system and has been demonstrated here for the first time in a vertebrate. It will now be possible to examine whether these identifiable glomeruli are functionally specialized in terms of odor processing. If so, zebrafish may emerge as a tractable model system for studies on olfactory coding.

[Key words: odorant encoding, vertebrate, fish, zebrafish, Brachydanio rerio, olfactory system, glomerulus, olfactory receptor, olfactory bulb, axon tracing, Dil, confocal microscopy J

The principles of odorant encoding by the olfactory system are still largely unknown. The olfactory glomeruli were suspected early on of serving as functional units of olfaction, mainly because of their discrete anatomical appearance (Allison and War-

\footnotetext{
Received Feb. 19, 1993; revised June 18, 1993; accepted June 29, 1993.

We thank Friedrich Bonhoeffer for generous support of this work. We are grateful to Thomas Voigt and Friedrich Bonhoeffer for their critical reviews of the manuscript and Rosemary Drescher for correcting the language. We also thank A. Egert, P. Haffter, M. Mullins, and S. Schulte-Merker for their kind advice in breeding and raising of zebrafishes, and J. Berger for introducing H.B. to confocal microscopy. H.B. is supported by a fellowship of the Boehringer Ingelheim Fonds.

Correspondence should be addressed to Herwig Baier, Max-Planck-Institut für Entwicklungsbiologie, P.O. Box 2109, D-72011 Tübingen, Germany.

Copyright (C) 1994 Society for Neuroscience $0270-6474 / 94 / 140219-12 \$ 05.00 / 0$
}

wick, 1949; Le Gros Clark, 1957). These spherical neuropil structures are a prominent and ubiquitous feature of the vertebrate olfactory bulb and represent the first relay stations in the olfactory pathway. Many axons of olfactory sensory neurons converge into single glomeruli. Each axon terminates within only one glomerulus and forms multiple synaptic contacts on dendritic arbors of second-order neurons (Allison and Warwick, 1949). Evidence for the functional-unit hypothesis has been obtained by activity mapping techniques, such as the 2-deoxyglucose method (e.g., Stewart et al., 1979; Lancet et al., 1982), and by electrophysiology (Leveteau and Mac Leod, 1966; Buonviso and Chaput, 1990; Imamura et al., 1992). These studies showed that responses to certain odorants can be localized to spatial units corresponding to single glomeruli.

Similar neuropil conglomerates, also called glomeruli, are found at comparable locations in the olfactory pathways of arthropods (e.g., Sandeman and Luff, 1973; Ernst et al., 1977) and mollusks (Chase and Tolloczko, 1986). In several insect orders, the whole set of glomeruli was mapped by serial reconstruction of tissue sections and found to form a constant pattern among individuals of the same species (Rospars and Chambille, 1981; Rospars, 1983; Stocker et al., 1983; Arnold et al., 1985; Rospars and Hildebrand, 1992). Single glomeruli were homologized according to their size, their shape, and their relative position within the glomerular array. In addition, the discovery of sexually dimorphic glomeruli in cockroaches and moths allowed to attribute pheromone signal-processing to neurons connected to those glomeruli (Boeckh and Boeckh, 1979; Christensen and Hildebrand, 1987; Hansson et al., 1992). These findings strengthen the notion that glomeruli are functional units at least in the invertebrate olfactory system.

Up to now, it has been unclear whether vertebrates exhibit a similar constancy of glomerular configuration. Experience-induced alterations in glomerular size and number seem to speak against this notion (Woo et al., 1987; Royet et al., 1989). On the other hand, in a few cases, distinct groups of glomeruli have been identified among individuals of a species in vertebrates (Jastreboff et al., 1984; Shinoda et al., 1987). However, in no case has this been possible for single glomeruli. Attempts to this end may have been impeded by the fact that the animal species commonly studied, namely, rat, mouse, rabbit, salamander, frog, trout, and goldfish, possess relatively large olfactory bulbs with many hundreds or thousands of glomeruli. Thus, stereotyped patterns may have been overlooked due to the sheer number of glomeruli.

We investigated glomerular variability in the olfactory system of a small teleost, the zebrafish (Brachydanio rerio). In this animal, adult body length is a few centimeters, and the olfactory 
bulbs are small, but well developed. Here, we report that the olfactory glomeruli of the zebrafish are arranged in a constant pattern. As in insects, single homologous glomeruli can be identified from animal to animal.

\section{Materials and Methods}

Animals. Adult zebrafishes were obtained either from a local supplier (Pelz Aquaristik, Bondorf, Germany) or from our own wild-type breeding colony. Fishes of the latter source were $6-8$ months old $(2.8 \mathrm{~cm}$ body length, neasured from the tip of the snout to the base of the tail). Those of the former source varied in age as judged by their different body lengths $(2.8-3.5 \mathrm{~cm})$. Males and females were distinguished by the presence of either testes or ovaries. Differently sized goldfishes (5-1 l $\mathrm{cm}$ body length) were obtained from Pelz Aquaristik.

Anterograde labeling of glomeruli. All surgical and tissue-handling steps were performed under a dissection microscope. Dil (1,1'-dioctadecyl-3,3,3', 3'-tetramethylindocarbocyanine perchlorate; Molecular Probes) was dissolved in dimethylformamide at $5 \mathrm{mg} / 100 \mu 1$. This stock solution was freshly diluted 1:100 in deionized water. In some cases, the solution had to be sonicated and centrifuged briefly in order to remove crystals that otherwise lead to heterogeneous staining. Alternatively, an oily, noncrystallizing analog of DiI, the monounsaturated $\Delta^{9}$-DiI (1,1'-dioleyl-3,3,3', $3^{\prime}$-tetramethylindocarbocyanine methanesulfonate; Molecular Probes; $2.5 \mathrm{mg} / 100 \mu \mathrm{l}$ in methanol), was used at a dilution of 1:50. Both dyes gave comparable results, with the latter eliminating the need for sonication and centrifugation, but apparently being less well retained in the membranes.

The DiI solution (up to $2 \mu \mathrm{l}$ ) was injected into each nasal cavity of a fish that was previously anesthetized with tricaine methanesulfonate (MS-222, Serva Heidelberg; $0.01 \%$ for zebrafish, $0.05 \%$ for goldfish) and left in place for 5-10 min. Fishes were kept immobilized by placement on a paper towel drenched with ice-cold water. For injection, fine, flexible pipette tips (GELoader tips, Eppendorf) were used because they ideally fit the nares and do not damage the olfactory epithelium when accidentally touching it. When put back into a water tank, fishes generally revived within the first minute. They were then kept in a dark room overnight. Longer tracing periods did not improve staining. After decapitation both olfactory bulbs together with the telencephalic hemispheres were carefully released from the skull in chilled phosphatebuffered saline (PBS). Olfactory epithelia were collected as well in order to control for successful staining. Tissues were fixed in $4 \%$ paraformaldehyde in $0.1 \mathrm{M}$ phosphate buffer ( $\mathrm{pH} 7.4$ ) overnight, embedded in $5 \%$ gelatin, and postfixed for an additional $1-3 \mathrm{~d}$. Since exactly reproducible and symmetrical sectioning planes were desired for comparisons between different animals, the olfactory bulbs (plus attached telencephalon) were oriented properly before the gelatin had congealed. Vibratome sections at a thickness of $100 \mu \mathrm{m}$ were mounted on glass slides and coverslipped in PBS. On average six horizontal sections were obtained from one pair of olfactory bulbs. These sections were investigated under a fluorescence microscope (Axiophot, Zeiss) with rhodamine optics and photographed (TMax 400, Kodak). Some specimens were further analyzed by confocal microscopy.

Confocal microscopy. One hundred micrometer slices were further sectioned optically by means of a confocal laser-scanning microscope (Bio-Rad MRC-600) equipped with an argon laser (peak excitation wavelength, $514 \mathrm{~nm}$ ). The confocal system was operated with an inverted microscope (IM-35, Zeiss). To obtain an image of both olfactory bulbs, a $10 \times$ objective lens (Zeiss Neofluar, or Wild Fluotar) was used. The Wild objective was preferred because of its higher numerical aperture (0.45 NA) compared to $0.3 \mathrm{NA}$ for the Zeiss. For imaging of one olfactory bulb, a $16 \times$ objective lens (Zeiss Neofluar; $0.5 \mathrm{NA}$ ) was used. Because of the intense fluorescence of Dil the confocal aperture could be closed near to minimum while still retaining sufficient brightness of the signal. Optical sections were obtained at a step size of 5 or $10 \mu \mathrm{m}$ and stored as a series of images on an optical disk. An important advantage of confocal sectioning over mechanical sectioning is that adjacent images can be digitally collapsed to generate a two-dimensional projection. This technique circumvents tedious recomposition of thin serial sections. The Bio-Rad software offers several algorithms for this purpose. Best results were obtained with the "maximum projection" mode. In this mode, the brightest pixel values within each of the 512 $\times 512$ pixel columns are taken for the final image. Photographs of images were taken from a black-and-white monitor on TMax 100 films (Kodak).

\section{Results}

Injection of a DiI solution into the nasal cavities of adult zebrafishes leads to staining of the olfactory epithelium and anterograde labeling of olfactory nerve terminals exclusively within the ipsilateral olfactory bulb. Axon terminals are segregated into spherical or ellipsoid neuropil compartments that are readily visible in a whole-mount preparation of the olfactory system (Fig. 1). We will refer to these structures as glomeruli in the following, since they resemble typical vertebrate glomeruli in shape, size, and radial position (e.g., Andres, 1970). No Dillabeled axons extend into the deeper layers of the olfactory bulb. Within the glomerular neuropil brightly fluorescent swellings of fibers are visible that may represent synaptic boutons. The majority of glomeruli are clearly distinguishable from their direct neighbors, even though some of them are contiguous or even confiuent with each other. The diameters of different glomeruli vary between 25 and $140 \mu \mathrm{m}$. In addition to discrete glomeruli, there is some unparcellated glomerular terminal plexus mainly in the anterior and lateral regions of the olfactory bulb, as is indicated in Figure 1.

The glomerular pattern of one ulfactory bulb is repeated in the contralateral bulb in a mirror-symmetric fashion down to the level of single glomeruli. This observation implies that glomeruli are orderly distributed over the bulbar surface. We therefore investigated whether the glomerular arrangements were similar for different animals. For such comparisons, about 100 olfactory bulbs were investigated, of which 20 (from five male and seven female animals) were analyzed in detail, either as single olfactory bulbs or as pairs, by confocal microscopy. By using this technique instead of conventional wide-ficld microscopy, the spatial resolution could be considerably improved, as illustrated by Figure 2. For evaluation of the glomerular pattern, glomeruli were subdivided into five groups, named dorsal, ventral, lateral, medial, and anterior group, according to their respective locations within the olfactory bulb. This served for unambiguous determination of positions in the glomerular layer. Though borders between these groups can be reproducibly drawn, it is uncertain whether they correspond to some intrinsic organizational principle. Single glomeruli were then mapped and their presence and appearance compared to those found at homotopic sites in different animals. Morphological criteria for individual glomeruli covered size, shape, and density of innervation, the latter being indicated by the relative intensity of anterograde labeling.

It was found that the overall pattern formed by the glomeruli is highly stereotyped. This is illustrated in Figure 3 by two representative series of images obtained from two animals, one male (Fig. $3 A, B$ ) and one female (Fig. $3 C, D$ ). Groups and subgroups of glomeruli appear unchanged in all olfactory bulbs investigated, with no major deviations in position and gross morphology. Most strikingly, several individual glomeruli could be identified among individuals: in all olfactory bulbs examined, we found homologous counterparts for a total of 22 glomeruli, which are marked by letters in Figure 3, $B$ and $C$, and are listed with their full names in Table 1 . A prominent example of an identified glomerulus is the ventroposterior glomerulus (vpG; see below). A summary of our analysis of the glomerular population is given in Table 1. Here, all elements of the glomerular array are listed, comprising both single glomeruli and glomerular plexus. The 22 individually identified glomeruli are specified morphologically in more detail, with respect to size and density 

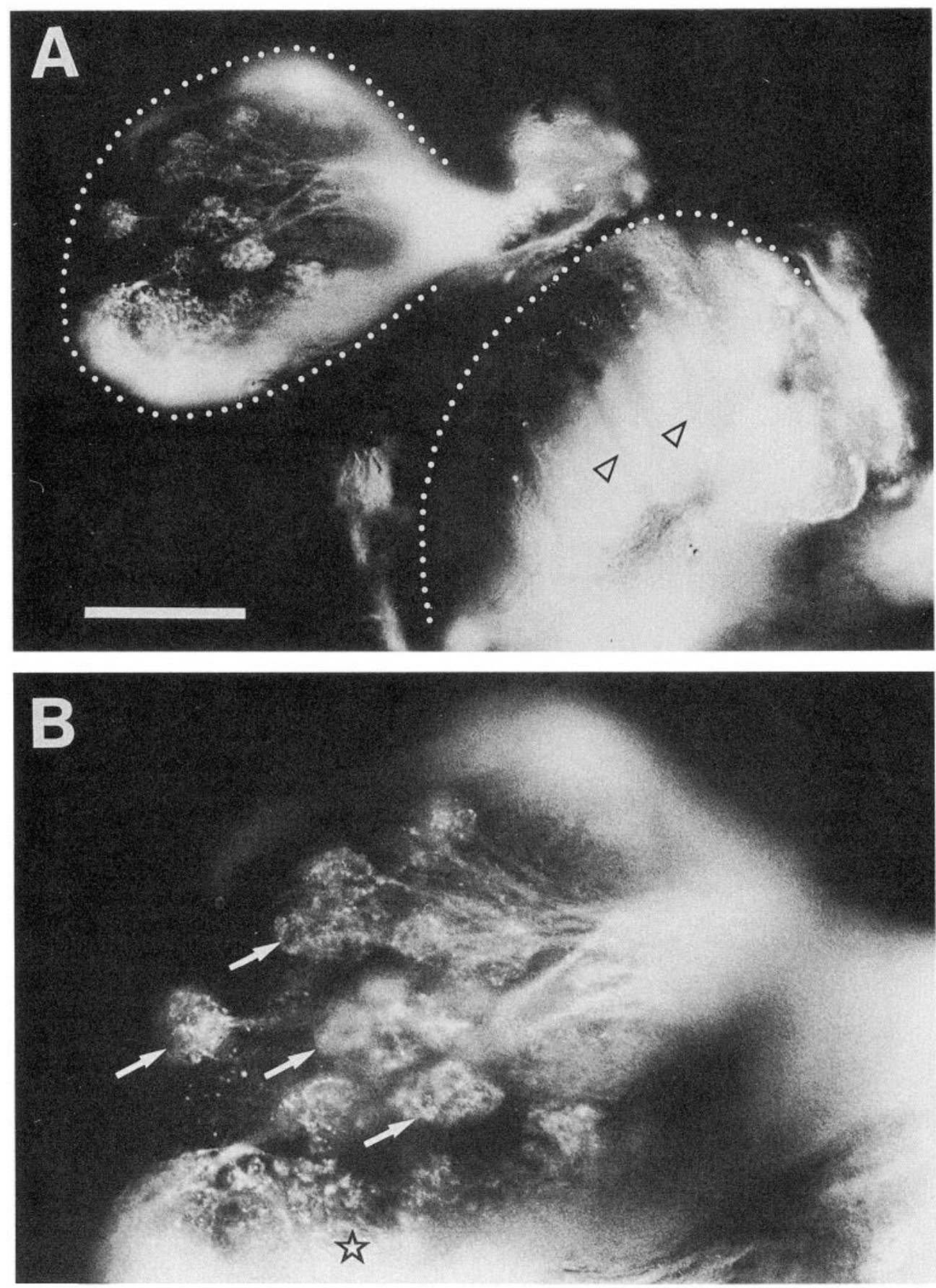

Figure 1. Zebrafish olfactory glomeruli stained anterogradely with DiI. $A$, Fluorescence photomicrograph of a whole-mount of the olfactory system, consisting of the cup-shaped olfactory epithelium (right) and the olfactory bulb (left), viewed from ventral. Both parts are outlined by dots. Medial is to the top, lateral to the bottom. A solution of DiI (bright fluorescence) was injected into the nasal cavity of the living fish. As a result, the olfactory epithelium is brightly stained, indicating that cells have taken up the dye and have incorporated it into their membranes. DiI is transported anterogradely within the axons of olfactory sensory neurons through the olfactory nerve (not visible) into the outer layers of the olfactory bulb. Within the olfactory epithelium, triangles mark borders between lamellae (a typical feature of the teleostean olfactory organ), which are hardly visible in this side view of the olfactory epithelium. $B$, Higher magnification of the olfactory bulb shown in $A$. Several glomeruli are visible (arrows). Star denotes unparcellated glomerular plexus in the lateral olfactory bulb. Scale bar, $250 \mu \mathrm{m}$ for $A, 125 \mu \mathrm{m}$ for $B$. of innervation. Several glomeruli (up to 10 per animal, often less) could not be consistently detected in all animals (for reasons discussed below) and are therefore named "unclassified" in Table 1 . In addition, as a third category, the list includes about 50 small glomeruli, all belonging to an anatomically well-defined cluster in the dorsal olfactory bulb. These glomeruli are morphologically homogeneous, relatively small $(25-30 \mu \mathrm{m}$ in diameter), densely packed, and arranged in several layers (Fig. 4). The dorsal cluster itself is always present at homotopic positions and contains a nearly constant number of glomeruli $(N \pm$ SEM $=49 \pm 1$, measured for 12 animals). Within this cluster, however, single glomeruli are indistinguishable. In addition to about 80 anatomically separable glomeruli ( 22 identified, less than 10 unclassified, and 49 dorsal cluster glomeruli), there exists unparcellated glomerular plexus, which is a well-known feature of teleosts (e.g., Kosaka and Hama, 1982). Though dominant in subregions of the olfactory bulb ("anterior plexus" and "plexus of the lateral chain"; see Table 1), glomerular plexus constitutes only a minor fraction of the total glomerular layer. Thus, even if the plexus contained additional "hidden" glomeruli, this would not essentially alter the total number of glomeruli.

We investigated whether systematic differences among the fishes studied, such as sex, age, or origin, can be correlated with differences in the pattern of glomeruli. For example, it is conceivable that sex-specific glomeruli exist, since olfactory cues trigger reproductive behaviors in zebrafish (Bloom and Perl- 

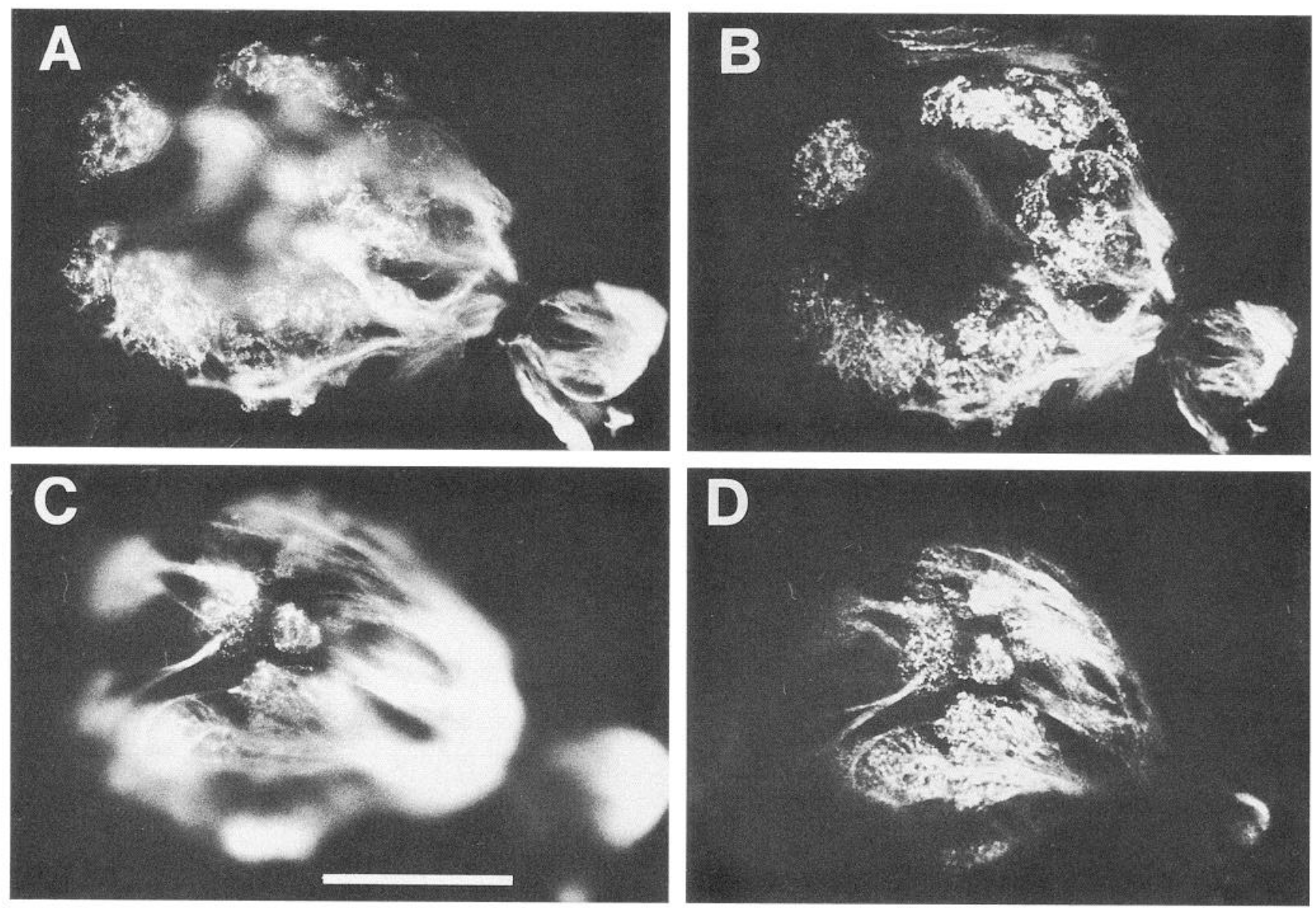

Figure 2. Comparison between pictures obtained by conventional wide-field microscopy $(A, C)$ and by confocal laser-scanning microscopy $(B$, $D)$. Pictures are taken from one and the same section at two different focal planes showing glomeruli in the ventral olfactory bulb. The olfactory nerve, fragments of which are visible, enters from the right (anterior). Medial is to the top. The spatial resolution is better for $B$ and $D$ than for $A$ and $C$, which correspond to the same focal planes, respectively, because out-of-focus blur is subtracted by confocal microscopy. Scale bar, $200 \mu \mathrm{m}$.

mutter, 1977; Van den Hurk and Lambert, 1983). We therefore examined male and female olfactory bulbs, separately. No sexual dimorphism in the glomerular pattern was detected (compare Fig. 3). We also failed to find systematic differences with respect to the presence of identified glomeruli between mature, but differently aged animals (as judged from their different body sizes). Furthermore, we compared the glomerular appearance in animals from our laboratory colony with that of animals obtained from a local fish supplier. This was done, first, to rule out that the stereotypy in the glomerular distribution was due to inbreeding and, second, to test whether it was a result of identical raising conditions. No differences were detected be- tween individuals raised at our institute and those from the alternative source. The latter population possessed heterogeneous genetic and geographic backgrounds. The finding that the glomerular pattern is constant and stable for all animals, irrespective of sex, age, and origin, suggests that it is formed by mechanisms that are common to all members of this species, and that it does not change notably with the ongoing growth of the adult organism.

Does the anatomical stereotypy extend to the morphological details of a single glomerulus? To test this, we selected one easily recognized glomerulus, the $\mathrm{vpG}$, and compared its contours in five pairs of olfactory bulbs (Fig. 5). It is found that shape and

Figure 3. Serial horizontal sections of two pairs of olfactory bulbs showing stereotyped distributions of glomeruli. All series start with the dorsalmost (row 1) and end with the ventralmost section (row 11). Anterior is to the bottom of each panel. $A$ and $D$, Confocal images obtained from a male $(A)$ and a female $(D)$ animal. Photographs do not represent single confocal planes, but stacked images of three to five adjacent optical sections, corresponding to a combined section thickness of 30-50 $\mu \mathrm{m}$. Marginal sections of the ventral and dorsal surface of the olfactory bulb were skipped because they contained solely olfactory nerve bundles and no glomeruli. $B$ and $C$, Drawings of sections shown in $A$ and $D$, respectively. The outlines of glomeruli and glomerular subgroups are depicted. Glomerular contours were drawn from highly magnified photographs. To obtain the best resolution available, the corresponding negatives were projected onto white sheets of paper. (This procedure is most convenient for the eyes since on negatives fluorescent structures appear dark on a white ground.) Broken lines indicate either just-emerging glomeruli, which are faintly visible in this section, or uncertain boundaries between contiguous glomeruli. For clarity, axon fascicles unrelated to glomerular structures have been omitted though they are visible on the corresponding photographs (see, e.g., D, row 11). Letter-code for identified glomeruli (for abbreviations, see Table 1): $a, \mathrm{dcaG}_{1} ; b, \mathrm{dcaG}_{2} ; c, \mathrm{dcaG}_{3} ; d, \mathrm{dcaG}_{4} ; e, \mathrm{dcaG}_{5} ; f, \mathrm{mdpG}_{1} ; g, \mathrm{mdpG}_{2} ; h, \mathrm{mdG} ; i, \mathrm{maG} ; k, \mathrm{meG}_{2} ;, \mathrm{lcG}_{1} ; m, \mathrm{lcG}_{2} ; n, \mathrm{lcG}_{3} ; o, \mathrm{lcG}_{4} ; p$,

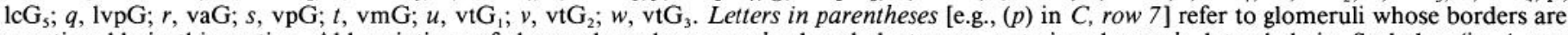
questionable in this section. Abbreviations of glomerular subgroups: $d c$, dorsal cluster; $a p$, anterior plexus; $l c$, lateral chain. Scale bar (in $A$, row 11), $250 \mu \mathrm{m}$ for all panels. 

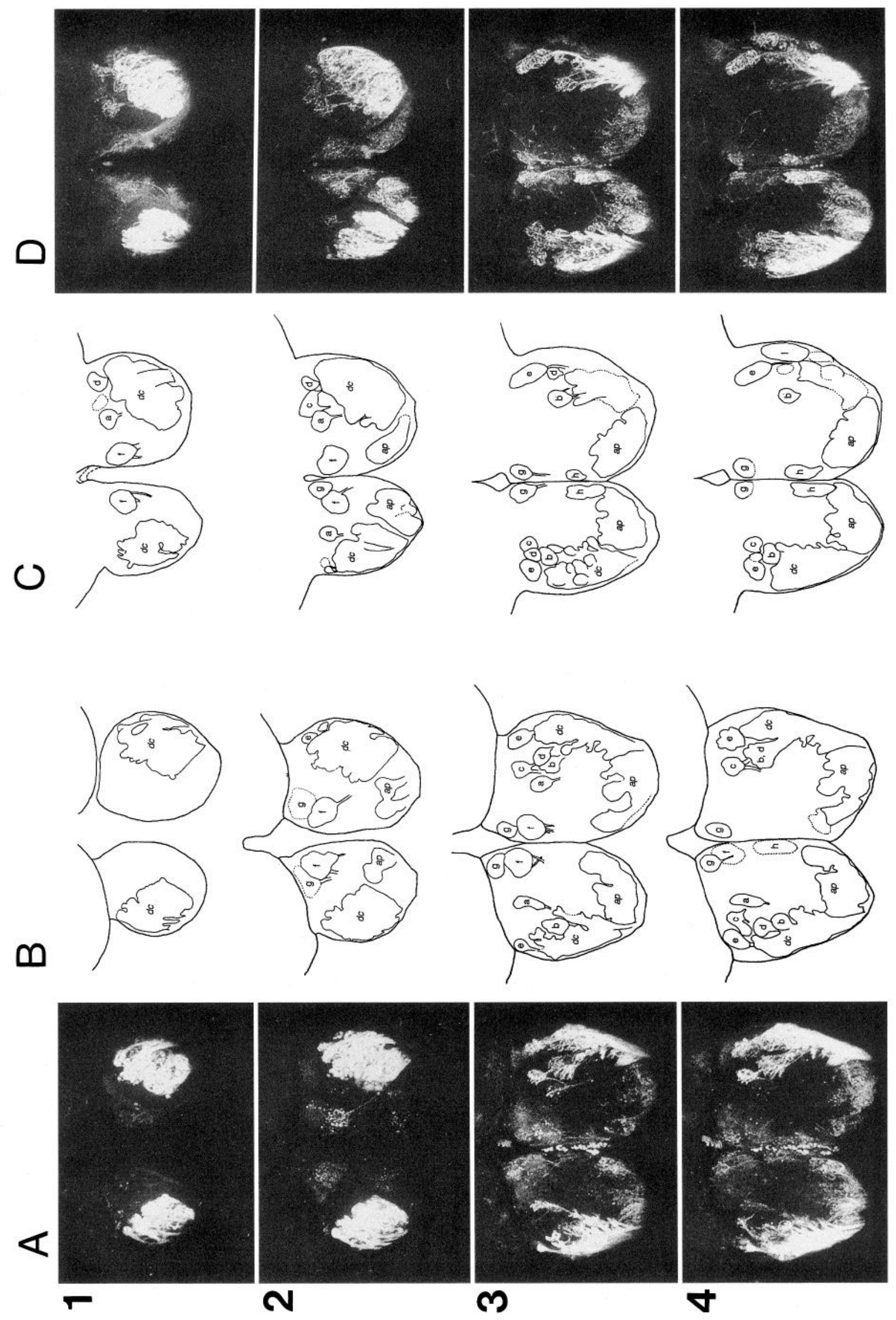

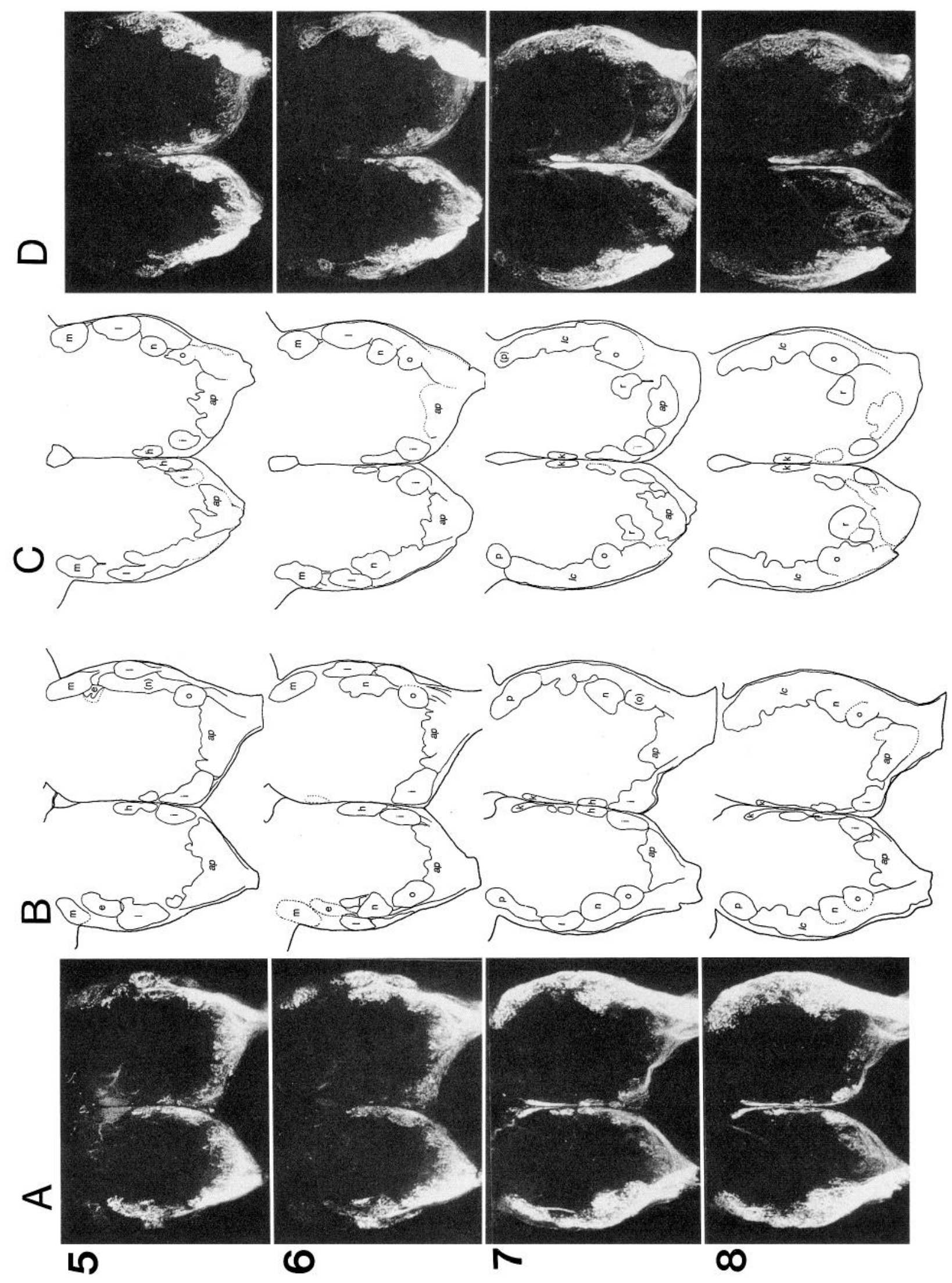

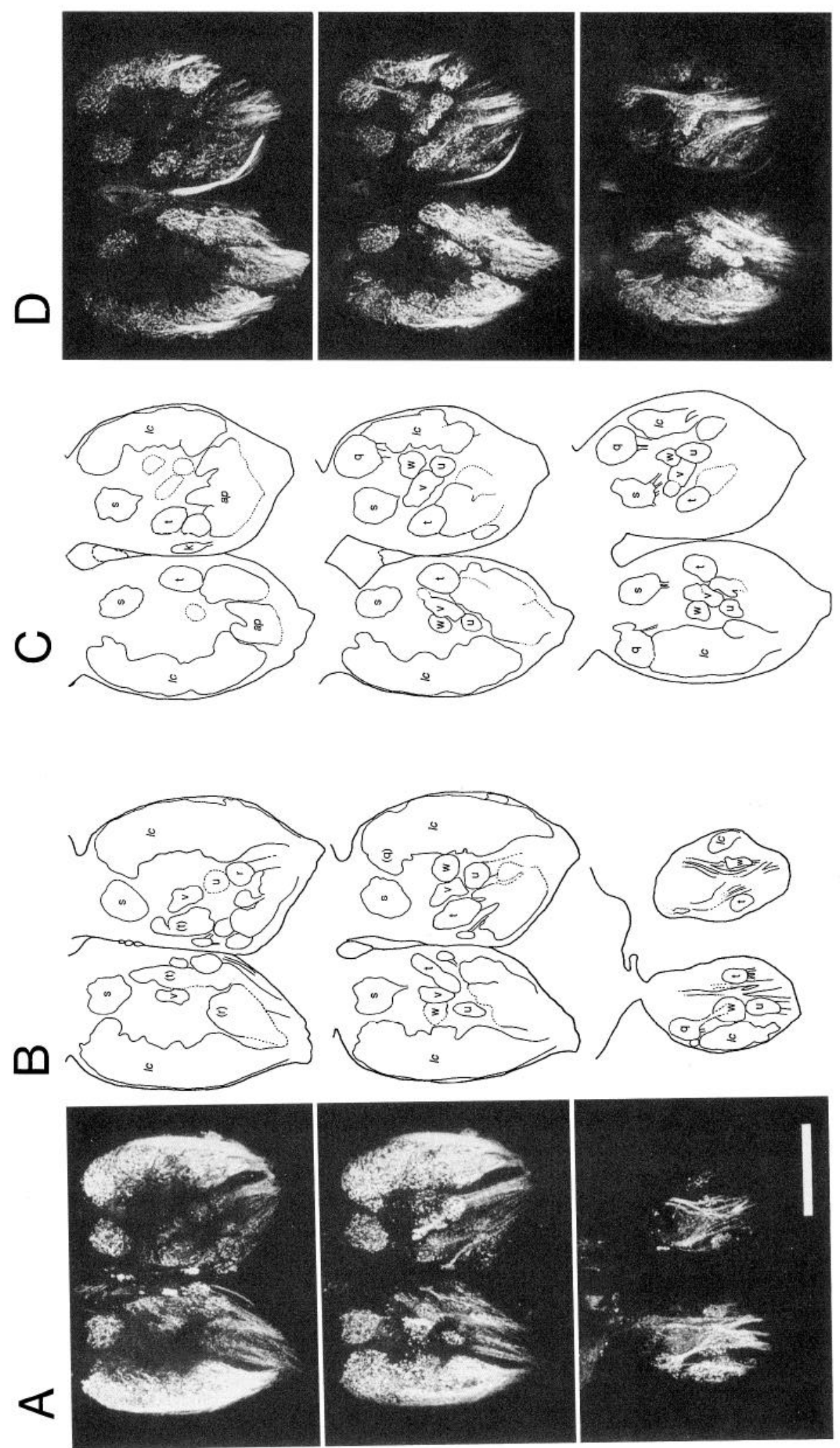

O)

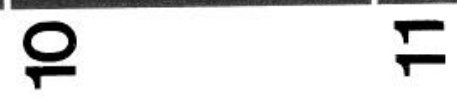




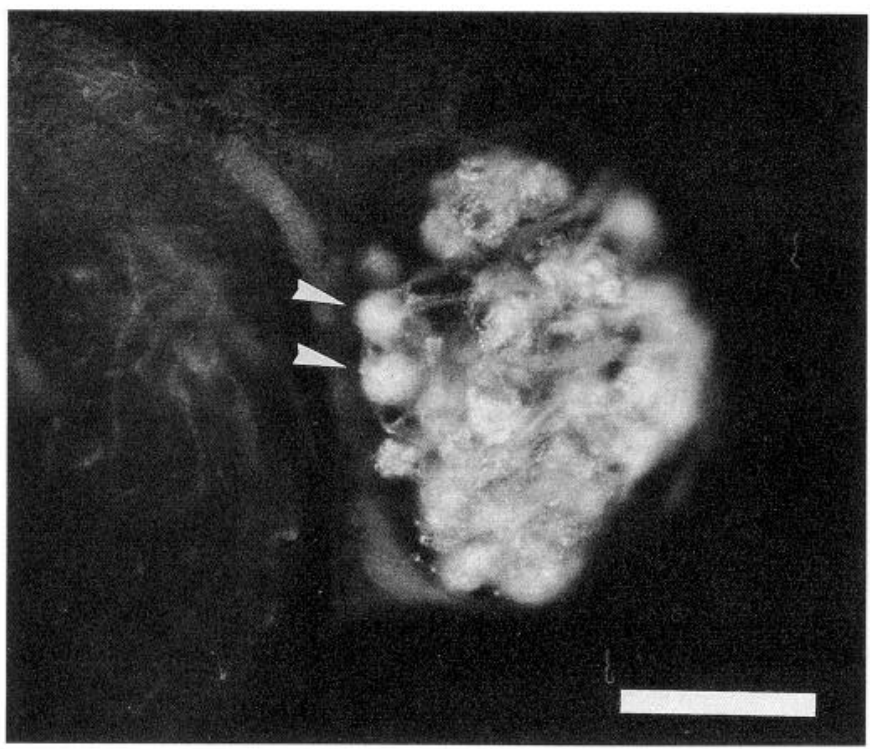

Figure 4. Glomeruli in the dorsal cluster. Glomeruli are small and densely clustered, but many of them can be delineated individually (arrowheads). Anterior is to the right, medial is top. Scale bar, $100 \mu \mathrm{m}$.

size of the vpG change considerably from animal to animal, while they are relatively constant for both bulbs of one animal. This variability was observed with other glomeruli, too. We also noticed that the distances between glomeruli and their relative positions may change. For example, the dorsal-cluster-associated glomeruli $\mathrm{dcaG}_{2}, \mathrm{dcaG}_{3}$, and $\mathrm{dcaG}_{4}$ sometimes form three discrete entities and sometimes are fused to a varying extent. Again, similarities were found to be more pronounced intraindividually than interindividually. From these qualitative observations, we conclude that the shape of an individual glomerulus is subject to variation, though its position is highly stereotyped.

Is glomerular invariance specific for the zebrafish among the vertebrates? One could argue that anatomical stereotypy would result from developmental constraints imposed on a size-restricted system as in this species. We therefore performed experiments on a larger teleost species, the goldfish. Additionally, the goldfish offers the opportunity to search for cross-species homologies since it is closely related to zebrafish (both are cyprinid fishes). The goldfish olfactory bulb is at least four times larger in diameter than that of the zebrafish and contains many more glomeruli, which are less well delineated than zebrafish glomeruli. Despite this, we found that the glomerular pattern in the goldfish was essentially bilaterally symmetrical within one individual (Fig. 6) and did not vary notably among the five animals investigated. Moreover, several subgroups of glomeruli previously identified in the zebrafish seemed to be also present in the goldfish, for example, the dorsal cluster and the glomerular plexus of the lateral chain. As in the zebrafish, the stereotypy seems to extend to single glomeruli: a single, solitary glomerulus in the ventroposterior region of the olfactory bulb (Fig. 6) is detectable in all animals investigated. This glomerulus resembles the vpG of the zebrafish with respect to position, absence of neighboring glomeruli, and relative size. Hence, it is conceivable that even single glomeruli may be conserved among different species. Though far from being exhaustive, these observations indicate that glomerular stereotypy is not an exclusive
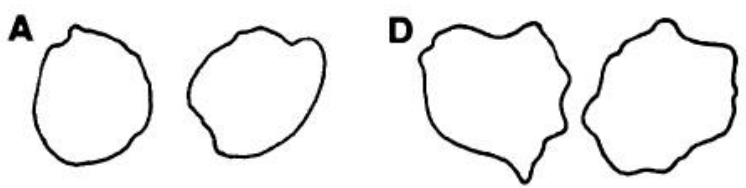

B
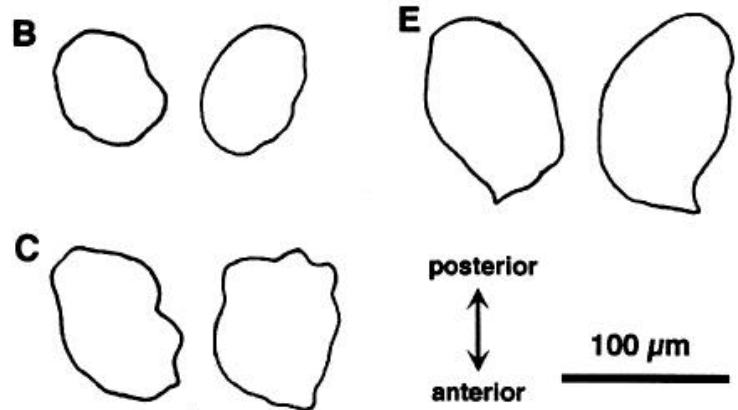

Figure 5. Contours of five pairs $(A-E)$ of ventroposterior glomeruli (vpG). Size and morphology of the vpG vary considerably interindividually, but tend to be similar intraindividually. Variations are not (or only to a minor extent) due to different sectioning angles, since the same identified glomeruli were always present in each of the sections from which the drawings were obtained.

feature of the zebrafish olfactory system, but also occurs in the larger, less compact system of the goldfish.

\section{Discussion}

We report here that the olfactory bulb of the zebrafish contains a small number of about 80 glomeruli, which are arranged in a stereotyped pattern. Twenty-two glomeruli could be recognized reliably from animal to animal as single morphological entities. This is the first description of single invariant glomeruli in a vertebrate. Earlier work on rodents has revealed that histologically identifiable groups of glomeruli exist in vertebrates (Greer et al., 1982; Jastreboff et al., 1984; Shinoda et al., 1984). For one such group, the so-called modified glomerular complex, a functional role in recognition of maternal pheromones by infants could be established (Greer et al., 1982). Our findings add the important aspect that the architecture of the glomerular population in a vertebrate can be highly stereotyped, down to the positions of individual glomeruli. This degree of stereotypy could not be predicted from previous studies on vertebrate olfaction.

Our report constitutes an interesting parallel to the insect olfactory system, in which invariant glomeruli have been described and studied extensively for several species, including moths (Rospars, 1983; Rospars and Hildebrand, 1992), cockroaches (Ernst et al., 1977; Rospars and Chambille, 1981), flies (Stocker et al., 1983), and bees (Arnold et al., 1985). In cockroaches and moths, specific glomeruli have been identified, which are involved in primary processing of sex pheromones (Boeckh and Boeckh, 1979; Christensen and Hildebrand, 1987; Hansson et al., 1992). This work has produced the best evidence available that single glomeruli serve as functional units for spatial encoding of odorant information (Hansson et al., 1992). By analogy, particular zebrafish glomeruli may process specific behaviorally relevant odors. Clearly, a decision on functional attributes of identified glomeruli has to await electrophysiological mapping of responses to defined odorants. In insects, an important functional difference between the male and the female olfactory system is reflected in the anatomy of the pheromone-selective glomeruli: they are well developed in males and comparably 


\begin{tabular}{|c|c|c|c|c|}
\hline $\begin{array}{l}\text { Regional group } \\
\text { Single glomeruli/subgroup }\end{array}$ & Short name ${ }^{h}$ & Width $(\mu \mathrm{m})$ & Length $^{c}(\mu \mathrm{m})$ & Innervation $^{d}$ \\
\hline \multicolumn{5}{|l|}{ Dorsal group } \\
\hline Glomeruli of the dorsal cluster $(49 \pm 1)^{\circ}$ & - & $25 \ldots .30$ & $25 \ldots .30$ & +++ \\
\hline \multirow[t]{5}{*}{ Dorsal-cluster-associated glomeruli (5) } & $\mathrm{dcaG}_{1}$ & $39 \pm 4$ & $49 \pm 5$ & ++ \\
\hline & $\mathrm{dcaG}_{2}$ & $39 \pm 2$ & $50 \pm 3$ & ++ \\
\hline & $\mathrm{dcaG}_{3}$ & $39 \pm 2$ & $48 \pm 3$ & ++ \\
\hline & $\mathrm{dcaG}_{4}$ & $36 \pm 3$ & $54 \pm 5$ & ++ \\
\hline & $\mathrm{dcaG}_{5}$ & $39 \pm 6$ & $99 \pm 10$ & ++ \\
\hline \multicolumn{5}{|l|}{ Medial group } \\
\hline \multirow[t]{2}{*}{ Mediodorsal posterior glomeruli (2) } & $\mathrm{mdpG}_{1}$ & $63 \pm 3$ & $72 \pm 4$ & + \\
\hline & $\mathrm{mdpG}_{2}$ & $40 \pm 3$ & $56 \pm 3$ & + \\
\hline Mediodorsal glomerulus & $\mathrm{mdG}$ & $45 \pm 2$ & $106 \pm 7$ & +++ \\
\hline Medioanterior glomerulus & $\mathrm{maG}$ & $53 \pm 4$ & $81 \pm 6$ & +++ \\
\hline Medial elongated glomerulus & $\mathrm{meG}$ & $15 \pm 1$ & $81 \pm 5$ & ++++ \\
\hline Unclassified glomeruli $(\max .4$ ) & - & - & - & - \\
\hline \multicolumn{5}{|l|}{ Anterior group } \\
\hline Anterior plexus & - & - & - & ++ \\
\hline Unclassified glomerulif (max. 2) & - & - & - & - \\
\hline \multicolumn{5}{|l|}{ Lateral group } \\
\hline \multirow[t]{5}{*}{ Glomeruli of the lateral chain (5) } & $\mathrm{lcG}_{1}$ & $45 \pm 3$ & $101 \pm 9$ & +++ \\
\hline & $1 c G_{2}$ & $50 \pm 5$ & $73 \pm 8$ & ++ \\
\hline & $\mathrm{lcG}_{3}$ & $59 \pm 3$ & $84 \pm 7$ & +++ \\
\hline & $\mathrm{lcG}_{4}$ & $61 \pm 5$ & $77 \pm 6$ & +++ \\
\hline & $\mathrm{lcG}_{5}$ & $51 \pm 3$ & $74 \pm 3$ & + \\
\hline Plexus of the lateral chain & - & - & - & +++ \\
\hline Lateroventral posterior glomerulus & lvpG & $69 \pm 6$ & $75 \pm 6$ & +++ \\
\hline Unclassified glomerulif (max. 1) & - & - & - & - \\
\hline \multicolumn{5}{|l|}{ Ventral group } \\
\hline Ventroanterior glomerulus & $\mathrm{vaG}$ & $51 \pm 3$ & $64 \pm 3$ & ++ \\
\hline Vcntroposterior glomerulus & $\mathrm{vpG}$ & $72 \perp 6$ & $102 \perp 8$ & ++ \\
\hline \multirow[t]{3}{*}{ Glomeruli of the ventral triplet (3) } & $\mathrm{vtG}_{1}$ & $48 \pm 3$ & $60 \pm 5$ & ++ \\
\hline & $\mathrm{vtG}_{2}$ & $44 \pm 2$ & $82 \pm 6$ & +++ \\
\hline & $\mathrm{vtG}_{3}$ & $48 \pm 2$ & $68 \pm 3$ & ++ \\
\hline Ventromedial glomerulus & $\mathrm{vmG}$ & $54 \pm 6$ & $81 \pm 8$ & ++ \\
\hline Unclassified glomerulif (max. 3) & - & - & - & - \\
\hline
\end{tabular}

${ }^{a}$ Gomeruli are listed as individuals if their presence was verified in all olfactory hulbs investigated $(N=20$; see Results). These glomeruli have been assigned names based on their positions within the olfactory bulb. Remaining glomeruli either are summarized as "unclassified" or belong to the dorsal cluster. The number of glomeruli in a specific subgroup is given in parentheses. For "unclassified" glomeruli the maximum number found in a single specimen is given. Presence of plexus is mentioned if it contributes significantly to the glomerular array (i.e., in the anterior and lateral group).

"Abbreviations for identified glomeruli.

-Shortest (width) and longest (length) diameter across the respective glomerulus. Values give the mean diameter of eight different olfactory bulbs in micrometers and the SEMs. Most glomeruli are ellipsoids, as can be judged from the ratio of "width" and "length."

"Innervation density was judged from the rclative brightness of labeling by visual inspection and was ranked on a subjective scale ranging from weakly stained $(t)$ to densely stained $(++++)$.

"Mean number \pm SEM. Glomeruli of the dorsal cluster were counted on images of 12 olfactory bulbs obtained with a $16 \times$ lens.

Glomeruli were considered "unclassified" if their presence and/or appearance was inconsistent for the olfactory bulbs investigated.

small in females. Although male zebrafish are attracted to sex pheromones released from the ovaries of females (Van den Hurk and Lambert, 1983; see below) - a behavior comparable to sex attraction in cockroaches and moths - no sexual dimorphism of glomeruli in male and female animals has been detected by us. Nevertheless, the analogies of the insect and the fish olfactory system suggest that the odor coding strategies of vertebrates and arthropods may be similar.

For several reasons, we do not think that the anatomical invariance observed here exclusively applies to the zebrafish among the vertebrates. (1) The olfactory system of the zebrafish conforms in its general features to the basic plan found throughout the vertebrates (Andres, 1970). In its special features, it represents a typical teleostean pattern (H. Baier and S. Korsching, unpublished observations; for review, see Yamamoto, 1982; Tucker, 1983). Furthermore, zebrafish glomeruli resemble ordinary vertebrate glomeruli in shape, size, radial position, and cellular composition (Baier and Korsching, unpublished observations; for review, see Andres, 1970). (2) Indications for a similar stereotypy in glomerular configuration were found by us 
Figure 6. Glomerular structures in a ventral section of the goldfish olfactory bulb. Anterior is to the bottom. A solitary glomerulus in the ventroposterior region found on both sides is indicated by arrowheads. This particular glomerulus was found in each animal investigated and may resemble the homolog of the zebrafish vpG (see Results). The section plane is somewhat tilted, so the medioventral glomeruli seen on the left side are not visible on the contralateral side (though they are present on the adjacent section, not shown). Note the presence of unparcellated lateral plexus on both sides that is also observed in the zebrafish. Scale bar, $200 \mu \mathrm{m}$.

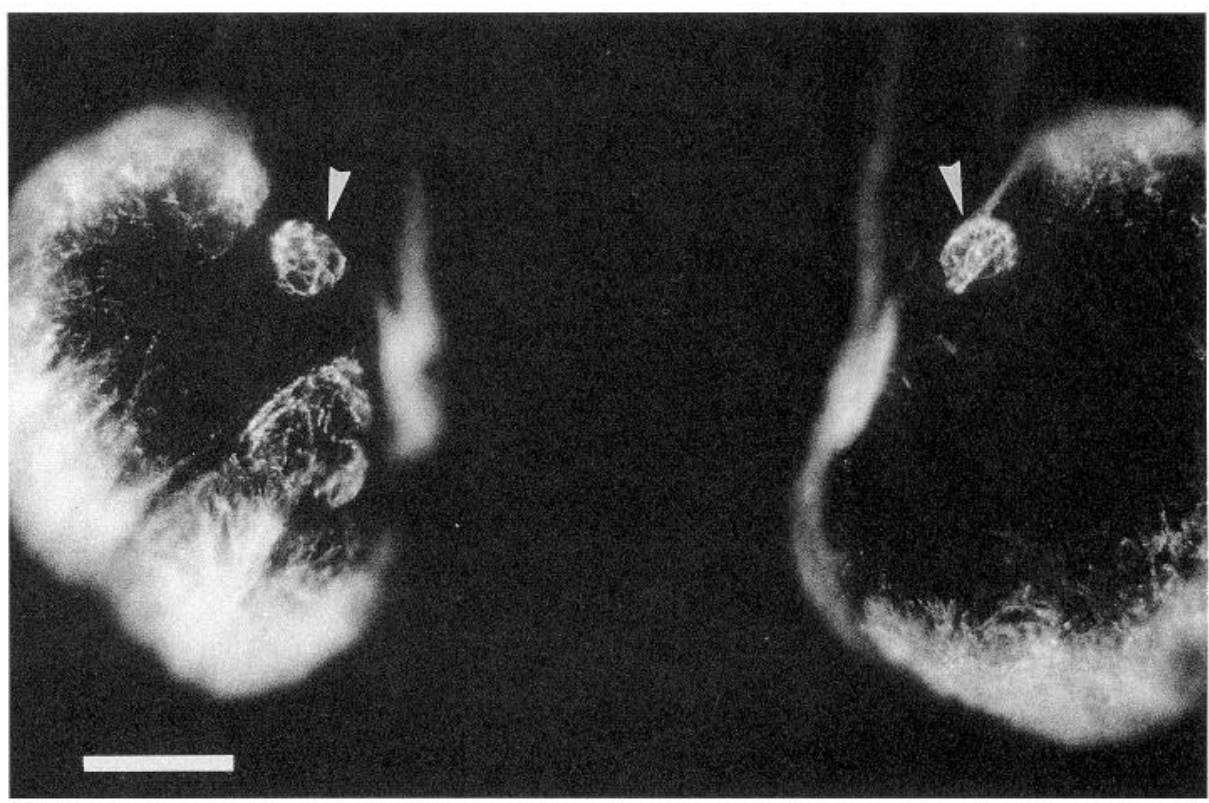

in another cyprinid species, the goldfish. A recent report on the trout, a salmonid teleost, documents strong similarities between individuals in the glomerular terminal fields (Riddle and Oakley, 1992). (3) In rodents, groups of glomeruli have been functionally and/or histologically identified at reproducible positions in the olfactory bulb (Greer et al., 1982; Jastreboff et al., 1984; Shinoda et al., 1987). Therefore, it seems unlikely that zebrafish represent an exotic case among the vertebrates. The various degrees of stereotypy found in different vertebrate species may be interpreted in two ways. It is possible that a continuum of stereotypy in glomerular position exists with a large proportion of identifiable glomeruli in the zebrafish and a small minority of them in other vertebrates, say rodents. Alternatively, it could be that stereotyped distributions of glomeruli are a general feature of vertebrates and that the degree of invariance has been underrated in previous investigations. A decision on this point is difficult to make on purely anatomical grounds, because the number of glomeruli in most species usually studied is one or two orders of magnitude larger than in the zebrafish. For example, adult mice possess about 1800 glomeruli (Royet et al., 1988; Pomeroy et al., 1990) and adult rabbits 6300 glomeruli (Royet et al., 1992) per olfactory bulb, compared to only 80 in the zebrafish.

While we were able to consistently identify 22 out of about 80 glomeruli in the zebrafish (i.e., $28 \%$ ), it is not yet decided whether the remaining glomeruli are truly variable. This is partly due to the limited histological resolution of single glomeruli in subregions of the olfactory bulb (mainly anterior and lateroventral). Here, glomerular plexus predominates over circumscribed glomeruli, the borders of which often cannot be delineated unequivocally. Consequently, we do not know whether a specific glomerulus observed in one animal is absent or simply not detectable in a different animal. An additional uncertainty with respect to single glomerulus identity applies to the glomeruli of the dorsal cluster, which contains more than half of the total population. These glomeruli are small and isomorphic. However, two observations suggest that the invariance may extend to a larger fraction of the glomerular population, rather than being restricted to the 22 identified glomeruli. First, the overall configuration of glomeruli is bilaterally symmetrical in a pair of olfactory bulbs even for glomeruli that are not well circumscribed and protrude only slightly from surrounding glomerular plexus. Second, although glomeruli of the dorsal cluster are indistinguishable from each other, their number is strikingly constant for all olfactory bulbs $(49 \pm 1)$. It is therefore conceivable that for any given glomerulus a homolog in a different animal exists. Alternatively, the stereotypy could be incomplete with an undetermined fraction of glomeruli (but less than $72 \%$ ) being variable.

One could argue that olfactory abilities are generally correlated with the number of glomeruli. According to a simplistic scheme, an animal with 10 times more glomeruli could smell 10 times better. Goldfish, for example, then should perform much better in olfaction than zebrafish, since the surface of their olfactory bulb is 10-20 times larger and therefore harbors many more glomeruli. Differences in olfactory acuity between these two species could refer to sensory detection thresholds, specificity of responses to chemically similar odorants, or accuracy in distinguishing complex mixtures of odorants. Whereas goldfish olfaction has been studied behaviorally and physiologically with a large number of odorants (for a recent report, see Zippel et al., 1993), to our knowledge, no such data exist for the zebrafish. Quantitative comparisons therefore are impossible to make. However, zebrafish respond to numerous olfactory cues by distinct, often stereotyped behaviors, like other teleosts (for review, see Little, 1983). In our laboratory, we have observed food-searching behavior and snapping when certain amino acids or food filtrates are presented, similar to reports on other teleosts. It is likely that these behaviors are for the most part elicited by olfaction (Little, 1983). We also have observed a teleosttypical fright reaction of zebrafish in response to alarm substances derived from the skin of conspecifics and in response to chemicals that elicit the fright reaction in related teleosts (Pfeiffer, 1978). A few studies exist on how sexual behaviors of zebrafish are triggered or inhibited by chemosensory stimuli: Chen and Martinich (1975) reported that males release a pheromone that induces females to ovulate. On the other hand, ovulation is repressed by metabolites in a crowded environment 
(Chen and Martinich, 1975). Bloom and Perlmutter (1977) demonstrated both inter- and intrasexual attraction in zebrafish by pheromones. The intrasexual attraction could be attributed to a cholesterol ester extracted from aquarium water (Algranati and Perlmutter, 1981). Males are attracted to females by a steroid glucuronide released from the ovaries (Van den Hurk and Lambert, 1983). These different types of behaviors, which are in no sense unique to the zebrafish, suggest that this species exhibits a well-developed and versatile sense of smell.

According to two extreme views, a reproducible pattern of glomeruli could either arise from a stereotyped development, or be shaped by similar environmental experiences. Since the animals employed in this study were raised under different conditions, one group in the laboratory colony, the other in the tanks of at least two commercial breeders, we assume that the contribution of experience to the stereotyped configuration of glomeruli is small, if at all present. Instead, intrinsic developmental mechanisms seem to underlie this process. If this is the case, it would be interesting to see whether the olfactory afferents organize themselves autonomously or whether the glomerular configuration is induced retrogradely by the olfactory bulb. Both possibilities are not necessarily exclusive. For example, in moth larvae, both afferent axons and glia cells in the target region are required for the assembly of glomeruli (Tolbert and Oland, 1989; Tolbert and Sirianni, 1990).

Unlike insects, vertebrates exhibit a life-long turnover of olfactory sensory neurons (Graziadei and Monti Graziadei, 1979). Additionally, in teleosts, the olfactory epithelium grows throughout adulthood together with the rest of the organism (Tucker, 1983). It follows that afferent fibers have to be continuously added and replaced within the glomeruli. Nevertheless, since individual glomeruli are present in the zebrafish at different life ages, as shown here, the population of glomeruli is apparently stable in the mature animal. This is consistent with the recent demonstration that, in mice, new glomeruli, once formed, persist to adulthood (LaMantia and Purves, 1989; Pomeroy et al., 1990). Because of their small number and known identities, zebrafish glomeruli may well be accessible to the investigation of mechanisms of module formation in the vertebrate brain that are still a subject of debate (Purves et al., 1992).

Organizing principles in the sense of smell, with respect to both developmental and functional aspects of the olfactory system, are still poorly understood. Many authors suggest that the glomeruli play an essential role in odorant encoding. Evidence for this, however, is still elusive. A system with a reduced number of glomeruli, a large fraction of which are individually identifiable, could be a valuable tool to solve this issue. We therefore propose that the zebrafish may constitute an excellent model for a systematic approach to these questions.

\section{References}

Algranati FD, Perlmutter A (1981) Attraction of zebrafish, Brachydanio rerio, to isolated and partially purified chromatographic fractions. Environ Biol Fish 6:31-38.

Allison AC, Warwick RTT (1949) Quantitative observations on the olfactory system of the rabbit. Brain 72:186-197.

Andres KH (1970) Anatomy and ultrastructure of the olfactory bulb in fish, amphibia, reptiles, birds, and mammals. In: Taste and smell in vertebrates (Wolstenholme GEW, Knight J, eds), pp 177-196. London: Churchill.

Arnold G, Masson C, Budharugsa S (1985) Comparative study of the antennal afferent pathway of the workerbee and the drone (Apis mellifera L.). Cell Tissue Res 242:593-605.
Bloom HD, Perlmutter A (1977) A sexual aggregating pheromone system in the zebrafish, Brachydanio rerio (Hamilton-Buchanan). J Exp Zool 199:215-226.

Boeckh J, Boeckh V (1979) Threshold and odor specificity of pheromone-sensitive neurons in the deutocerebrum of Antheraea pernyi and A. polyphemus (Saturnidae). J Comp Physiol [A] 132:235-242.

Buonviso N, Chaput MA (1990) Response similarities to odors in olfactory bulb output cells presumed to be connected to the same glomerulus: electrophysiological study using simultaneous single-unit recordings. J Neurophysiol 63:447-454.

Chase R, Tolloczko B (1986) Synaptic glomeruli in the olfactory systcm of a snail, Achatina fulica. Cell Tissuc Res 246:567-573.

Chen L, Martinich RL (1975) Pheromonal stimulation and metabolite inhibition of ovulation in the zebrafish, Brachydanio rerio. Fishery Bull 73:889-894.

Christensen TA, Hildebrand JG (1987) Male-specific, sex pheromoneselective projection neurons in the antennal lobes of the moth Manduca sexta. J Comp Physiol [A] 160:553-569.

Ernst KD, Boeckh J, Boeckh V (1977) A neuroanatomical study on the organization of the central antennal pathways in insects. II. Deutocerebral connection in Locusta migratoria and Periplaneta amaericana. Cell Tissue Res 176:285-308.

Graziadei PPC, Monti Graziadei GA (1979) Continuous nerve cell renewal in the olfactory system. In: Handbook of sensory physiology, Vol IX (Jacobson M, ed), pp 55-83. Berlin: Springer.

Grccr CA, Stewart WB, Teicher TH, Shepherd GM (1982) Functional development of the olfactory bulb and a unique glomerular complex in the neonatal rat. J Neurosci 2:1744-1759.

Hansson BS, Ljungberg H, Hallberg E, Löfstedt C (1992) Functional specialization of olfactory glomeruli in a moth. Science $256: 1313-$ 1315.

Imamura K, Mataga N, Mori K (1992) Coding of odor molecules by mitral/tufted cells in rabbit olfactory bulb. I. Aliphatic compounds. J Neurophysiol 68:1986-2002.

Jastreboff PJ, Pedersen PE, Greer CA, Stewart WB, Kauer JS, Benson TE, Shepherd GM (1984) Specific olfactory receptor populations projecting to identified glomeruli in the rat olfactory bulb. Proc Natl Acad Sci USA 81:5250-5254.

Kosaka T, Hama K (1982) Structure of the mitral cell in the olfactory bulb of the goldtish. J Comp Neurol 212:365-384.

LaMantia AS, Purves D (1989) Development of glomerular pattern visualized in the olfactory bulbs of living mice. Nature 341:646-649.

Lancet D, Greer CA, Kauer JS, Shepherd GM (1982) Mapping of odor-related neuronal activity in the olfactory bulb by high-resolution 2-deoxyglucose autoradiography. Proc Natl Acad Sci USA 79:670674.

Le Gros Clark WE (1957) Inquiries into the anatomical basis of olfactory discrimination. Proc R Soc Lond [Biol] 146:299-319.

Leveteau J, Mac Leod P (1966) La discrimination des odeurs par les glomérules olfactifs du lapin (Etude electrophysiologique). J Physiol (Paris) 58:717-729.

Little EE (1983) Behavioral function of olfaction and taste in fish. In: Fish neurobiology (Northcutt RG, Davis RE, eds), pp 351-375. Rexdale: Wiley Canada.

Pfeiffer W (1978) Heterocyclic compounds as releasers of the fright reaction in the giant danio Danio malabaricus (Jerdon) (Cyprinidae, Ostariophysi, Pisces). J Chem Ecol 4:665-673.

Pomeroy SL, LaMantia A-S, Purves D (1990) Postnatal construction of neural circuitry in the mouse olfactory bulb. J Neurosci 10:19521966.

Purves D, Riddle DR, Pomeroy SL (1992) Iterated patterns of brain circuitry (or how the cortex gets its spots). Trends Neurosci 15:362368.

Riddle DR, Oakley B (1992) Immunocytochemical identification of primary olfactory afferents in rainbow trout. J Comp Neurol 324: $575-589$.

Rospars JP (1983) Invariance and sex-specific variations of the glomerular organization in the antennal lobes of a moth, Mamestra brassicae, and a butterfly, Pieris brassicae. J Comp Neurol 220:8096.

Rospars JP, Chambille I (1981) The deutocerebrum of the cockroach Blaberus cruniifer Burm: quantitative study and automated identification of glomeruli. J Neurobiol 12:221-247. 
Rospars JP, Hildebrand JG (1992) Anatomical identification of glomeruli in the antennal lobes of the male sphinx moth Manduca sexta. Cell Tissue Res 270:205-227.

Royet JP, Souchier C, Jourdan F, Ploye H (1988) Morphometric study of the glomerular population in the mouse olfactory bulb: numerical density and size distribution along the rostrocaudal axis. J Comp Neurol 270:559-568

Royet JP, Jourdan F, Ploye H, Souchier C (1989) Morphometric modifications associated with early sensory experience in the rat olfactory bulb. II. Stereological study of the population of olfactory glomeruli. J Comp Neurol 289:594-609.

Royet JP, Hudson R, Distel H, Meyer F (1992) Is the ratio number of mitral cells per glomerulus constant? Xth Congress of ECRO Abstracts, p 133

Sandeman DC, Luff SE (1973) The structural organization of glomerular neuropile in the olfactory and accessory lobes of an Australian freshwater crayfish, Cherax destructor. Z Zellforsch 142:37-61.

Shinoda K, Shiotani Y, Osawa Y (1989) "Necklace olfactory glomeruli" form unique components of the rat primary olfactory system. J Comp Neurol 284:362-373.

Stewart WB, Kauer JS, Shepherd GM (1979) Functional organization of the rat olfactory bulb analyzed by the 2-deoxyglucose method. $J$ Comp Neurol 185:715-734.
Stocker RF, Singh RN, Schorderct M, Siddiqi O (1983) Projection patterns of different types of antennal sensilla in the antennal glomeruli of Drosophila melanogaster. Cell Tissue Res 232:237-248.

Tolbert LP, Oland LA (1989) A role for glia in the development of neuropilar structures. Trends Neurosci 12:70-75.

Tolbert LP, Sirianni PA (1990) Requirement for olfactory axons in the induction and stabilization of olfactory glomeruli in an insect. $J$ Comp Neurol 298:69-82.

Tucker D (1983) Fish chemoreception: peripheral anatomy and physiology. In: Fish neurobiology (Northcutt RG, Davis RE, eds), pp 311 349. Rexdale: Wiley Canada.

Van den Hurk R, I ambert JGD (1983) Ovarian steroid glucuronides function as sex pheromones for male zebrafish, Brachydanio rerio. Can J Zool 61:2381-2387.

Woo CC, Coopersmith RM, Leon M (1987) Localized changes in olfactory bulb morphology associated with early olfactory learning. $J$ Comp Neurol 263:113-125.

Yamamoto $M$ (1982) Comparative morphology of the peripheral olfactory organ in teleosts. In: Chemoreception in fishes (Hara TJ, ed), pp 39-59. Amsterdam: Elsevier.

Zippel HP, Voigt R, Knaust M, Luan Y (1993) Spontaneous behaviour, training and discrimination in goldfish using chemosensory stimuli. J Comp Physiol [A] 172:81-90. 